\title{
Abrasiveness Calibration of Optical Measuring Wheel
}

\author{
J.Y. Shieh, W.C. Huang, Y.T. Liu, G.J. Liao \\ Department of Electro-Optics Engineering \\ National Formosa University \\ Yunlin County, 632, Taiwan
}

\begin{abstract}
The aim of this study is to propose a simple operation and highly practical measuring instrument. In response to conventional mechanical measuring wheels that mainly measure distance mechanically and inspect wheel wear visually, however, they are unable to monitor it effectively. As the wheel tends to generate wheel problems over time, it will gradually cause inaccurate distance measurement. Thus, a Holtek microcontroller HT66F50 was adopted to allow it to have a measuring function by coordinating with an infrared ranging module and an incremental encoder. Besides, Measurement System Analysis was further conducted to verify whether or not this measuring system is able to comply with the measurement system specifications.
\end{abstract}

Keywords-measuring wheel; wheel wear; microcontroller; infrared ranging module for distance measurement; encoder

\section{INTRODUCTION}

As time progresses, many types of distance measuring devices have been marketed to use extensively in our daily life. Among the general measuring tools available are tape measures, measuring wheels, electronic distance measuring instruments, etc. As the conventional measuring wheels only inspect wheel wear visually, they are unable to monitor it effectively. To date, no products are available to monitor the wheel condition on the measuring wheels. As the conventional measuring wheels are functioned mechanically, the longer they have been used, the greater they will generate distance measuring error due to the abrasion problem on the wheel.

\section{LITERATURE REVIEW}

In the chapter, infrared sensors and encoders will be discussed. Then, the extensive applications of infrared sensors and encoders will be learned.

\section{A. Infrared}

Infrared sensors have been conducted by many researchers. A simple infrared sensor is generally composed of a transmitter and a receiver, and the distance calculation of an infrared sensor between the sensor and target is determined by the signal strength emitted by the transmitter to the receiver. Infrared sensors are widely applied in robots [1-3]. As for automated equipment, it is applied in target detecting to check whether or not an object has come near [4, 5]. Other applications include non-onsite monitoring and detection of pest amount [6]. In addition, the use of infrared sensors can be also widely found in program controls [7-9], security systems, distance and depth monitoring, ground sensors, position measurements and controls [10], obstacle collision avoidance [11], and map establishment [12,13].

Principles of electronic distance measurement are mainly the beam modulation and the pulse-echo method.
1) The Beam modulation method: The beam modulation method is also known as the phase difference method, which uses the phase difference between the reflected wave and reference wave caused by irradiation of electronic signals on an object. The transmitter will first emit a modulated beam, and if the phase while the receiver is capturing the Echo Signal is referred to as $\varphi$, then the Phase Meter will be able to detect the phase difference between the signal transmission and receiving. This has been used to calculate the distance as shown in Fig. 1.

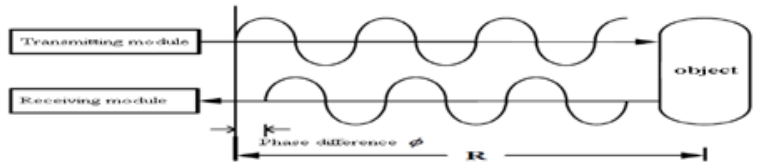

FIGURE I. BEAM MODULATION FOR A DISTANCE MEASUREMENT.

2) The pulse-echo method: The pulse-echo method uses an emitter to transmit a short and strong pulse. Through the distance traveled by this pulse and reflected by the object to the measuring system, the distance between the measuring system and the object can be obtained by calculating the time difference between the pulse transmission and receiving.

Fig. 2. shows a distance measurement with the pulse-echo method. The pulse is being emitted by a transmission module towards an object. The pulse signal reflected by it can be captured at the receiving end, and the distance can then be calculated based on the time needed for the pulse to travel back and forth.

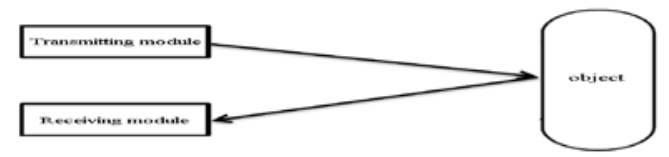

FIGURE II. A DISTANCE MEASUREMENT WITH THE PULSE-ECHO METHOD.

\section{B. Optical Encoders}

A ruler, vernier caliper and other measuring tools are used to measure distance. A ruleris a relatively simple tool to determine the length and is likely to cause errors. A Vernier caliperis scale that indicates where the measurement lies in between two of the marks on the main scale. It has a fixed scale and a sliding scale. Users can read the left-most tick mark on the sliding scale from the fixed scale. By adding these two measurements, the length of the object can be obtained. Although it is able to enhance accuracy, but the measuring range is small. Thus, the technical advancement has generated a new type of digital measuring tool known as the encoder . 
An Encoder is a one of digital measuring tools and the distance or angle measured is represented by symbols 1 and 0 . Most encoders are incorporated with an optical sensor, comprising of the basic elements, a light emitting diode (LED) or other light sources to emit the light, light-transmission grating, and a phototransistor placed in relative position of the LED. Encoders have many types; it can be classified by the operation principle or measurement object of which can be divided into optical, magnetic, inductive and capacitive types. Besides, if based on the calibration and signal output form of an encoder, it can also be divided into incremental and absolute types.

\section{BASIC STRUCTURE OF THE DEVICE}

As shown in Fig. 3, the microcontroller will capture the value from the infrared ranging module and transmit it back to MCU to do the calculation. It uses I/O Port both as the LCM signal transmission channel and the user control button to switch to transmission channel.

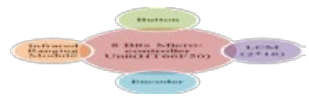

FIGURE III. BASIC STRUCTURE OF THE 8 BITS MICROCONTROLLER UNIT (HT66F50) DEVICE.

Generally, a measuring instrument is a device for measuring a physical quantity. Thus, the measuring instrument adopted in the study equips with an infrared ranging module, an encoder, a LCD module (LCM), and a8 bits microcontroller unit, shown in Fig. 4.

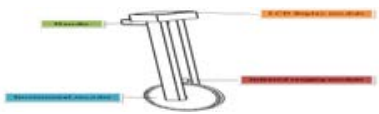

FIGURE IV. BASIC STRUCTURE OF THE DEVICE.

\section{Measurement Principle}

The incremental grating disc adopted in the study has a fixed diameter of $16.5 \mathrm{~cm}$. An infrared ranging module was used to measure the distance from the infrared end to the wheel, shown in Fig.5. When the wheel is used over time, its diameter will gradually become smaller due to wear. As the wheel measures the distance in a circular manner, and that the location of infrared ranging module placed by this study could only detect a single wheel surface, so the wheel is wearing off twice as much as its diameter. On the contrary, the distance detected by the infrared ranging module will become greater and greater. Hence, the wheel wear can then be measured.

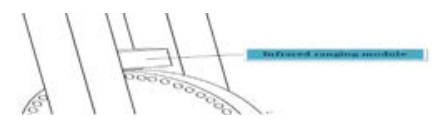

FIGURE V. INSTALLATION POSITION OF THE INFRARED RANGING MODULE.

The measurement principle of this study is to use the diameter to calculate the circumference as shown in equation (1).

$$
\mathrm{L}=\mathrm{R} \times \pi
$$

$\mathrm{L}$ is referred to as the circumference; $\mathrm{R}$ as the wheel diameter; and $\pi$ is equal to 3.14 .

45 gratings are on the incremental grating disc, so the length of a single grating can be obtained by dividing (1) with the total number of gratings as shown in equation (2).

$$
\mathrm{M}_{0}=\frac{\mathrm{L}}{\mathrm{M}}
$$

M0 is referred to as one grating; and $\mathrm{M}(45)$ as the total number of gratings. After calculating one grating, the distance measured is shown in equation (3).

$$
\mathrm{D}_{2}=\mathrm{M}_{0} \times \mathrm{M}_{1}
$$

D2 is referred to as the distance measured by this study; and M1 as the grating number that has traveled during the measurement.

Fig. 6. is the distance measured. The distance is calculated from grating between $\mathrm{A}$ and $\mathrm{B}$.

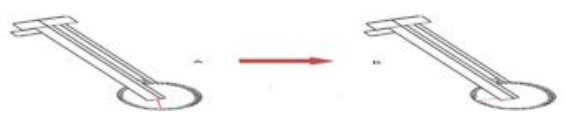

FIGURE VI. THE DISTANCE FROM GRATING BETWEEN A AND B.

\section{The Flow Chart of the Device}

To correct wheel wear and measure the distance, it is needed to know the integrated value of the infrared ranging module and the encoder value and then to do the calculation. The flow chart of the device is shown in Fig. 7.

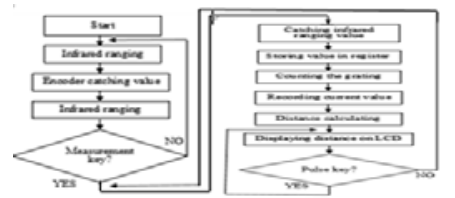

FIGURE VII. THE FLOW CHART OF THE DEVICE.

\section{RESUlTS OF THE STUDY}

In the actual measuring, the ceramic tile pavement on the floor is used as the tested object. Each square tile measures 30x30 cm (one grid). Measurements were done from 1 to 10 tiles, respectively. While measuring wheel wear, as it could not be known the immediate wear on the wheel, the wheel was initially bonded with $0.1,0.2$ and $0.3 \mathrm{~cm}$ of materials to ease measuring.

\section{A. Measurements for Wheel Wear}

In the actual measuring, the ceramic tile pavement on the floor is used as the tested object. Each square tile measures $30 \times 30 \mathrm{~cm}$ (one grid). Measurements were done from 1 to 10 tiles, respectively. While measuring wheel wear, as it could not be known the immediate wear on the wheel, the wheel was initially bonded with $0.1,0.2$ and $0.3 \mathrm{~cm}$ of materials to ease measuring.

1) $0.1 \mathrm{~cm}$ wheel wear: Followed up were the measurements of $0.1 \mathrm{~cm}$ wheel wear. Table 1 is the average value and error rate of 10 measurements of $0.1 \mathrm{~cm}$ wheel wear. The error rate between the average distance measured and actual value is about $1 \%$. 
TABLE I. THE ERROR RATE IN 0.1 CM WHEEL WEAR.

\begin{tabular}{|c|c|c|c|}
\hline $\begin{array}{c}\text { Numbers } \\
\text { of Tiles }\end{array}$ & & $\begin{array}{c}\text { Average Distance } \\
\text { Measured } \\
(\mathrm{cm}) \\
\end{array}$ & $\begin{array}{c}\text { The Error } \\
\text { Rate } \\
(\%) \\
\end{array}$ \\
\hline 1 & & 30.3 & 1.00 \\
\hline 2 & & 60.51 & 0.85 \\
\hline 3 & & 90.98 & 1.09 \\
\hline 4 & & 120.17 & 0.14 \\
\hline 5 & & 150.38 & 0.25 \\
\hline 6 & & 180.37 & 0.21 \\
\hline 7 & & 210.94 & 0.45 \\
\hline 8 & & 240.36 & 0.15 \\
\hline 9 & & 270.82 & 0.30 \\
\hline \multirow[t]{2}{*}{10} & & 301.91 & 0.30 \\
\hline & $\begin{array}{l}1.50 \% \\
1.00 \% \\
0.50 \% \\
0.00 \%\end{array}$ & The Error Rate in 0.1 wheet Wear & gria(s) \\
\hline
\end{tabular}

FIGURE VIII. THE DIAGRAM FOR THE ERROR RATE IN 0.1 WHEEL WEAR.

\section{B. MSA Analysis Report}

For the sake of Gauge Repeatability and Reproducibility, or GR\&R, Based on [14], the researcher believes that the analytical method of GR\&R measurement system in QS9000 can be used by any hardware manufacturing industries as QS9000, ISO9001 and ISO9002 all belong to a quality management system. Based on Table 4, the R\&R values of grating ranging wheel used by the study in measuring the corrected wheel wear are all less than $10 \%$; thus, it verifies that the measurement results of the study can be accepted by QS9000.

TABLE II. : \%R\&R INDICATOR.

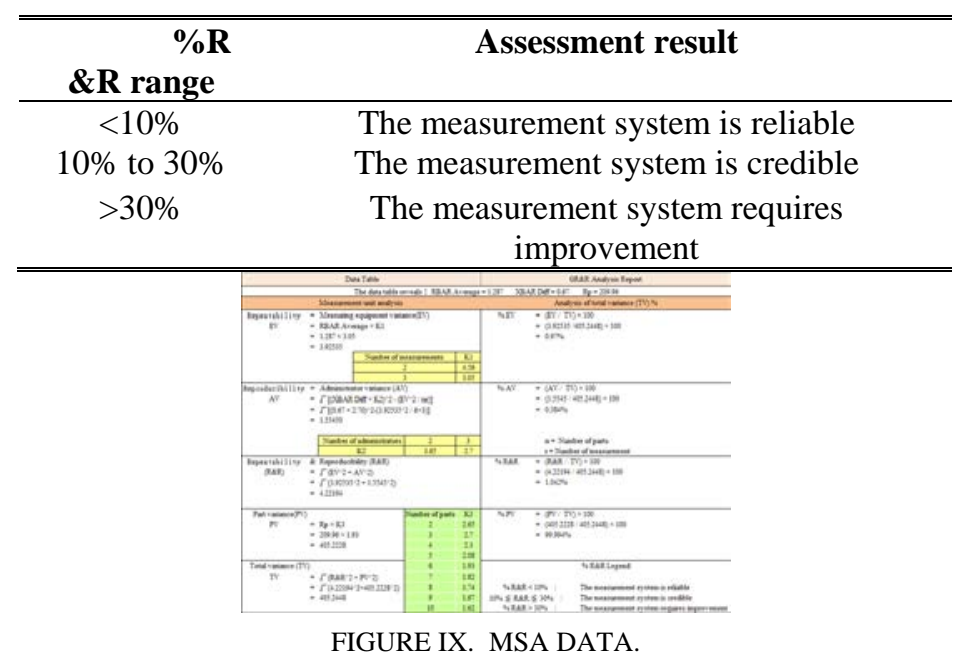

\section{CONCLUSIONS}

In this experiment, the greater the distance of uncorrected distance measures, the bigger the error rate will have. Since the study adopted the grating ranging wheel with corrected wheel wear to measure distance, and also took wheel wear into consideration; thus, the greater the distance measured, the smaller the error rate would have.

\section{REFERENCES}

[1] Guan-Jie Lyu, A study on the Design and Control of Line Tracking and Obstacle Avoidance for a Mobile Robot, TaTung University, 2012.

[2] Li T.-H.S, Shih-Jie Chang and Wei Tong, Fuzzy Target Tracking Control of Autonomous Mobile Robots by Using Infrared Sensors, IEEE Transactions on Fuzzy System, Vo1.12,mo.4,2004

[3] I-hsiang Lai, The following robot with searching and obstacle-avoiding, National Central University, 2009.

[4] Deividson L. Okopnik ,Rosane Falate, Usage of the DFRobot RB-DFR49 Infrared Sensor to detect maize seed passage on a conveyor belt, Computers and Electronics in Agriculture (2014) 106-111

[5] E. Cheung, V.J. Lumelsky, Proximity sensing in robot manipulator motion planning: system and implementation issues, IEEE Trans. Robotics Autom. 5 (6) (1989) 740-751.

[6] A.J. Hand, Infrared sensor counts insects, Photonics Spectra 32 (11) (1998) 30-31.

[7] G. Benet, F. Balnes, J.E. Simo, P. Perez, Using infrared sensors for distance measurement in mobile robots, Robotics and Autonomous Systems 40(2002) 255-266.

[8] B. Gopalakrishnan, S. Tirunellayi, R. Todkar, Design and development of an autonomous mobile smart vehicle: a mechatronics application, Mechatronics 14 (2004) 491-514.

[9] T. Aytac, B. Barshan, Differentiation and localization of targets using infrared sensors, Optics communications 210 (2002) 25-35.

[10] B. Butkiewicz, Position control system with fuzzy microprocessor AL220, Lecture Notes in Computer Science, vol. 1226, 1997, pp. 74-81.

[11] V.J. Lumelsky, E. Cheung, Real-time collision avoidance in teleoperated whole-sensitive robot arm manipulators, IEEE Trans. Syst. Man Cybern. 23 (1) (1993) 194-203.

[12] H.-H. Kim, Y.-S. Ha, G.-G. Jin, A study on the environmental map building for a mobile robot using infrared range-finder sensors, in:Proceedings IEEE/RSJ International Conference on Intelligent Robots and Systems, Las Vegas, NV, USA, 27-31 October 2003, pp. 711-716.

[13] M. Alwan, M. B Wagner, G. Wasson, P. Sheth, Characterization of infrared range-finder PBS-03JN for 2-D mapping, Robotics and Automation, ICRA 2005. Proceedings of the 2005 IEEE International Conference on 2005.

[14] Chiao-Yu Chiang, A Study of Gauge Repeatability and Reproducibility for Measurement Systems, National Cheng Kung University, 2002. 